\title{
High prevalence of vitamin D insufficiency in community-dwelling postmenopausal Polish women
}

\author{
Artur Stolarczyk ${ }^{1}$, Andrea Horvath ${ }^{2}$, Monika Szczechura ${ }^{1}$, Magda Kamińska $^{1}$, Piotr Dziechciarz ${ }^{2}$ \\ ${ }^{1}$ Clinical Rehabilitation Department, Medical University of Warsaw, Poland \\ 2Department of Paediatrics, Medical University of Warsaw, Poland
}

\begin{abstract}
Introduction: Inadequate vitamin D level is associated with altered bone turnover and bone loss, which increases the fracture risk.

Aim: To assess the seasonal prevalence of inadequate (insufficient or deficient) serum vitamin D levels in community-dwelling postmenopausal Polish women screened for osteoporosis.

Material and methods: A cross-sectional observational study based on the regional urban non-institutionalized sample $(n=107)$ of postmenopausal Caucasian women in the age range of 51-83 years, not taking any medication and free from any condition likely to affect vitamin D status or calcium/bone metabolism. The outcome measures were the mean $25-\mathrm{OH}$ vitamin $\mathrm{D}$ level across all the seasons and the percentage of vitamin $\mathrm{D}$ deficiency and insufficiency and defined as < $20 \mathrm{ng} / \mathrm{dl}(50 \mathrm{nmol} / \mathrm{l})$ and 20-30 ng/dl (50-75 nmol/l), respectively.

Results: No statistically significant difference has been found in the mean vitamin D level, regardless of the season $(p=0.4)$. The prevalence of vitamin D deficiency and insufficiency were in spring $54 \%$ and $32 \%$, in summer $46 \%$ and $46 \%$, in autumn $67 \%$ and $27 \%$, and in winter $61 \%$ and $22 \%$, respectively.

Conclusions: Vitamin D inadequacy is common in a sample of Polish community-dwelling postmenopausal women regardless of the season.
\end{abstract}

Key words: osteoporosis, cross-sectional study, screening.

\section{Introduction}

It has been postulated that vitamin D plays an active role not only in calcium/phosphate homeostasis and proper bone metabolism, but also in the prevention of cancer, autoimmune conditions, cardiovascular disease, infections, cognitive decline and a number of other conditions [1]. Although vitamin D can be delivered with diet, the number of foods naturally containing vitamin $D$ in significant amounts is very limited. Levels of circulating 25 -hydroxyvitamin $\mathrm{D}-25(\mathrm{OH}) \mathrm{D}$ - are largely determined by skin production that occurs in response to the solar UVB radiation [2, 3].

However, at latitudes above $33^{\circ}$, UV-B radiation is only effective in activating vitamin $D$ production during the summer months at the time of the midday [4]. In recent decades, several lifestyle changes, such as time spent indoors, sun avoidance due to skin cancer prophylaxis, the topical application of a sunscreen and increased pollution contribute to vitamin D inadequacy in European countries including Poland [5]. In 2013, the Central European group of experts have established recommendations for prophylactic vitamin D supplementation which advised universal supplementation of 800-2000 IU/day of vitamin D in patients over 65 years of age, throughout the year [6]. In adults below 65 years, the total supplementation of vitamin D should be provided between September and April, with a dose of 800-2000 IU/day and throughout the whole year in the case of inadequate vitamin D skin synthesis during the summer time. However, recommendations were based on expert opinions supported by studies performed in other countries because data regarding the vitamin D status across various age groups of the Polish population is still very limited [7].

The aim of the study was to evaluate the level of vitamin $D$ at different times of the year in the community-dwelling outpatient sample of postmenopausal women.

\section{Material and methods}

This study was a cross-sectional observational study based on the regional urban non-institutionalized sample of postmenopausal Caucasian women, over 50 years of age. The study took place in Warsaw, Poland $\left(52.26^{\circ} \mathrm{N}\right)$ between 1.01.2009 and 30.05.2010. 
The primary outcome measure was the mean $25(\mathrm{OH}) \mathrm{D}$ level across all the seasons (winter: January - March, spring: April - June, summer: July - September, autumn: October - December). The secondary endpoints were the percentage of vitamin D insufficiency and deficiency in the studied group and odds ratio of being sufficient or deficient with vitamin D in the postmenopausal women aged less than 65 years compared to older ones.

Patients referred to the outpatient clinic for initial screening for osteoporosis were invited to participate into the study. Women were consecutively enrolled, with no influence on the referral by any of the researchers. Women were eligible for this study if they had been postmenopausal for at least 1 year. The exclusion criteria were previous therapy for osteoporosis, renal, hepatic, or bowel dysfunction (inflammatory bowel disease and malabsorption), diabetes mellitus type 1 or 2 and uncontrolled thyroid or parathyroid gland dysfunction. After receiving information from the investigator, and being able to ask questions regarding any aspect of the study, all participants provided written informed consent before enrolment. Blood samples were obtained after overnight fasting between $8 \mathrm{AM}$ and noon. Samples were centrifuged (1800 g, $10 \mathrm{~min}$, room temperature), separated and stored at $-20^{\circ} \mathrm{C}$ until analysed.

The serum 25(OH)D level was measured by chemiluminescence immunoassay with standard kits (LIAISON ${ }^{\circledR}$, DiaSorin Inc., Stillwater, United States; inter-assay precision $5 \%$, intra-assay precision $7 \%$ ). For group discrimination, we used $20 \mathrm{ng} / \mathrm{ml}$ as the critical value for deficiency and $30 \mathrm{ng} / \mathrm{ml}$ as the cut-off value for insufficiency. The values over $30 \mathrm{ng} / \mathrm{ml}$ were considered to be the optimal $25(\mathrm{OH}) \mathrm{D}$ level according to expert recommendations.

In all patients, dual energy X-ray absorptiometry examination was performed using the LUNAR Prodigy (GE-Lunar Corp., Madison, Wisconsin, USA) densitometer. Bone mineral density (BMD) was measured at L2L4 lumbar spine in women below 65 years and femur neck over 65 years. The coefficient of variation of the technique at our laboratory was $0.8 \%$ using the anatomical spine phantom measure daily. Bone mineral density was expressed as $T$-score, a number of standard deviations from the mean of young women attaining peak bone mass. We considered osteoporosis when the lumbar spine or femur neck $T$-score was equal or below -2.5 standard deviation.

The study was accepted by the Ethics Committee at the Medical University of Warsaw.

\section{Statistical analysis}

Descriptive statistics are presented as mean and standard deviation (SD), as applicable. Categorical variables were expressed as a number of subjects and percentage with $95 \%$ confidence intervals $(95 \% \mathrm{Cl}) \cdot \chi^{2}$ test was used to compare the groups of categorical variables. One-way analysis of variance (ANOVA) was used for season group comparison. A value of $p<0.05$ was considered statistically significant.

\section{Results}

Of potentially 135 eligible women, altogether 115 patients participated in the study. One hundred and seven blood samples - 28 obtained in spring, 26 in summer, 30 in autumn, 23 in winter - were finally analysed. Eight blood samples were not analysed because of either loss or improper storage conditions. The group characteristics (age, BMI, \% of patients with osteoporosis) are shown in Table I.

There has been no statistically significant difference found in the mean vitamin D level regardless of the season (SS: 366,367, df: 3, MS: 128,789, F: 0.985, p: 0.403) (Table II). Altogether 12 (11\%) participants showed vitamin D sufficiency and 61 (57\%) demonstrated vitamin D deficiency according to predefined criteria (Table II). Only small percentages of patients $(6-17 \%$ of patients depending on the season) had the adequate serum level of 25(OH)D. Compared to younger women, patients $>65$ years had a statistically significant increased odds ratio for vitamin $\mathrm{D}$ deficiency $(7.6,95 \% \mathrm{Cl}: 21-47.1)$ and decreased OR for vitamin sufficiency $(0.16,95 \% \mathrm{Cl}$ : 0.04-0.68) in summer with no difference found in other seasons (data not shown).

\section{Discussion}

The results of our study show than there has been no seasonal pattern in the mean $25(\mathrm{OH}) \mathrm{D}$ level in the

Tab. I. Group characteristics

\begin{tabular}{lcccc}
\hline & $\begin{array}{c}\text { Spring } \\
n=29\end{array}$ & $\begin{array}{c}\text { Summer } \\
n=31\end{array}$ & $\begin{array}{c}\text { Autumn } \\
n=31\end{array}$ & $\begin{array}{c}\text { Winter } \\
n=24\end{array}$ \\
\hline Mean age \pm SD (years) & $71.4 \pm 8.7$ & $70.6 \pm 7.9$ & $70.0 \pm 7.0$ & $71.6 \pm 6.6$ \\
\hline Mean body mass index \pm SD & $29 \pm 4.2$ & $28.7 \pm 3.8$ & $28.3 \pm 3.2$ & $29.2 \pm 4.1$ \\
\hline \% of patients with osteoporosis & 81 & 77 & 77 & 84 \\
\hline $\boldsymbol{N}(\%)$ of patients $>65 \mathrm{y}$ & $22(76 \%)$ & $23(74 \%)$ & $21(68 \%)$ & $20(83 \%)$ \\
\hline
\end{tabular}


Tab. II. Vitamin D supply by season

\begin{tabular}{lccccc}
\hline & $\begin{array}{c}\text { Number } \\
\text { of patients }\end{array}$ & $\begin{array}{c}\text { Mean 25(OH)D level } \\
\pm \text { SD }(\mathrm{ng} / \mathrm{ml})\end{array}$ & $\begin{array}{c}\text { Vitamin D deficiency } \\
\%(n) \text { of pts }\end{array}$ & $\begin{array}{c}\text { Vitamin D insufficiency } \\
\%(n) \text { of pts }\end{array}$ & $\begin{array}{c}\text { Vitamin D suficiency } \\
\%(n) \text { of pts }\end{array}$ \\
\hline Spring & 28 & $18.6 \pm 10.5$ & $54 \%(15)$ & $32 \%(9)$ & $14 \%(4)$ \\
\hline Summer & 26 & $21.6 \pm 11.0$ & $46 \%(12)$ & $46 \%(12)$ & $8 \%(2)$ \\
\hline Autumn & 30 & $17.6 \pm 8.5$ & $67 \%(20)$ & $27 \%(8)$ & $6 \%(2)$ \\
\hline Winter & 23 & $21.3 \pm 15.2$ & $61 \%(14)$ & $22 \%(5)$ & $17 \%(4)$ \\
\hline
\end{tabular}

outpatient population of Polish postmenopausal women. Only approximately $11 \%$ of participants showed vitamin D sufficiency, whereas $57 \%$ of the analysed group showed deficiency defined as $25(\mathrm{OH}) \mathrm{D}$ level less than $20 \mathrm{ng} / \mathrm{ml}$.

Although serum $25(\mathrm{OH}) \mathrm{D}$ is the foremost indicator of vitamin D status, much debate surrounds the biochemical definition of adequacy of vitamin $D$ in the general population. Various thresholds for vitamin $D$ adequacy have been used. We assumed the 25(OH)D threshold of at least $30 \mathrm{ng} / \mathrm{ml}$ as optimal fracture prevention based on 2010 International Osteoporosis Foundation position statement on vitamin D [8], the 2011 US Endocrine Society Task Force on Vitamin D [9], European Menopause and Andropause Society position statement released in 2012 [10] and Central European recommendations [6].

As there is no consensus on the cut-offs used to identify the adequate vitamin D level and assays used for the 25(OH)D assessment differs among studies, making it difficult to compare results, our data are similar to the results of other European studies [11-16]. However in most but not in all [17] of them a significant seasonal variation has been noted. The lack of the increase in vitamin $D$ level in our population in the summer period seems to be attributed to culture-driven sun avoidance behaviour, clothing habits, scarce outdoor activities, or age-related decrease of vitamin D skin synthesis [18].

There is evidence that the elderly have one-fourth to one-fifth of the ability to make vitamin D from UV irradiation compared with adults younger than 30 years of age [19]. The results of our study suggest that in younger postmenopausal women, summer sun exposure seems to play a larger role in the prevention of vitamin $D$ deficiency. Participants $<65$ years have a significantly decreased odds ratio for being vitamin insufficient in summer but not in the other seasons compared to patients above that age. We did not measure vitamin $D$ intake in our patients, nevertheless some studies suggest that ageing populations, have a greater dependency on dietary sources to maintain optimal vitamin $D$ status [20, 21].

Recently published national representative food consumption data obtained from individuals showed that in $56 \%$ of Polish women $>60$ years dietary intake of vitamin $D$ is below the lower reference nutrient intake and almost all of them (96\%) did not meet estimated average reference values [22]. In Polish diet, very few foods contain an appreciable amount of vitamin D, margarine is the only food mandated to be fortified with vitamin $D$ and due to national eating habits, fish consumption frequency is still very low [23]. Population studies have reported higher rates of bone remodelling and fragility fracture during the winter, which suggests that any period of vitamin D insufficiency is unsuitable $[24,25]$. Thus, vitamin D deficiency throughout the year may increase the age-related bone loss leading to the earlier osteoporosis [26]. In our population, 4/5 of patients have osteoporosis, however because of the crosssectional study design, conclusion on the relationship between vitamin D status and bone mineral density is not possible.

These results should be interpreted in the light of the study's strengths and limitations. The study was based on a relatively small number of included females ( $n=107)$. Thus, the results of our study should be viewed with caution. Moreover, it may be also argued that the patients included in the present study may not be representative for the entire population of Polish postmenopausal women. Women referred for osteoporosis screening might have been more self-aware or might have been considered to be at greater risk by their general practitioner of the risks of bone-calcium metabolism. However, referral was based on the postmenopausal status only, and since we did not influence referral, we regard the study population as randomly selected.

The results of the study might be biased because our laboratory was not a part of the international vitamin D assays quality assessment scheme (DEQAS) which, would have enhanced the comparability of the results with those performed by other laboratories and assay methods. We did not assess vitamin D oral intake, so based on the results of this study we cannot estimate relative contribution of diet in vitamin $D$ supply. However, in the light of the fact that in Poland there is no universal vitamin D food fortification policy and typical Polish diet is not abundant in vitamin D, this study supports the need for universal supplementation in women. 
Based on the results of our study we may speculate that vitamin D through the year supplementation policy should be expanded to all postmenopausal women in Poland. Nevertheless, an adequately powered community based study analysing vitamin $D$ status in postmenopausal women aged less than 65 years is expected to provide a better answer for this assumption.

\section{Conclusions}

Vitamin D insufficiency is prevalent regardless of the season in an outpatient, community-dwelling sample of postmenopausal women living in Warsaw, Poland.

\section{Disclosure}

\section{Authors report no conflicts of interest.}

\section{References}

1. Holick MF. Vitamin D: extraskeletal health. Endocrinol Metab Clin North Am 2010; 39: 381-400

2. Holick MF. Vitamin D deficiency. N Engl J Med 2007; 357: 266-281.

3. Mithal A, Wahl DA, Bonjour JP, IOF Committee of Scientific Advisors (CSA) Nutrition Working Group. Global vitamin D status and determinants of hypovitaminosis D. Osteoporos Int 2009; 20: 1807-1820.

4. Engelsen 0 . The relationship between ultraviolet radiation exposure and vitamin D status. Nutrients 2010; 2: 482-495.

5. Moan J, Baturaite Z, Juzeniene A, et al. Vitamin D, sun, sunbeds and health. Public Health Nutr 2012; 15: 711-715.

6. Płudowski P, Karczmarewicz E, Bayer M, et al. Practical guidelines for the supplementation of vitamin $D$ and the treatment of deficits in Central Europe - recommended vitamin D intakes in the general population and groups at risk of vitamin D deficiency. Endokrynol Pol 2013; 64: 319-327.

7. Napiórkowska L, Budlewski T, Jakubas-Kwiatkowska W, et al. Prevalence of low serum vitamin D concentration in an urban population of elderly women in Poland. Pol Arch Med Wewn 2009; 119: 699-703.

8. Dawson-Hughes B, Mithal A, Bonjour JP, et al. IOF position statement: vitamin $D$ recommendations for older adults. Osteoporos Int 2010; 21 : 1151-1154.

9. Holick MF, Binkley NC, Bischoff-Ferrari HA, et al. Endocrine Society. Evaluation, treatment, and prevention of vitamin D deficiency: an Endocrine Society clinical practice guideline. J Clin Endocrinol Metab 2011; 96: 1911-1930.
10. Pérez-López FR, Brincat M, Erel CT, et al. EMAS position statement: Vitamin D and postmenopausal health. Maturitas 2012; 71: 83-88.

11. Burnand B, Sloutskis D, Gianoli F, et al. Serum 25-hydroxyvitamin D: distribution and determinants in the Swiss population. Am J Clin Nutr 1992; 56: 537-542.

12. Scharla SH, Scheidt-Nave C, Leidig G, et al. Lower serum 25-hydroxyvitamin $D$ is associated with increased bone resorption markers and lower bone density at the proximal femur in normal females: a populationbased study. Exp Clin Endocrinol Diabetes 1996; 104: 289-292.

13. Meyer HE, Falch JA, Søgaard AJ, et al. Vitamin D deficiency and secondary hyperparathyroidism and the association with bone mineral density in persons with Pakistani and Norwegian background living in Oslo, Norway, The Oslo Health Study. Bone 2004; 35: 412-417.

14. Lamberg-Allardt CJ, Outila TA, Kärkkainen MU, et al. Vitamin D deficiency and bone health in healthy adults in Finland: could this be a concern in other parts of Europe? J Bone Miner Res 2001; 16: 2066-2073.

15. Kudlacek S, Schneider B, Peterlik M, et al. Austrian Study Group on Normative Values of Bone Metabolism. Assessment of vitamin D and calcium status in healthy adult Austrians. Eur J Clin Invest 2003; 33 323-331.

16. MacFarlane GD, Sackrison JL Jr, Body JJ, et al. Hypovitaminosis D in a normal, apparently healthy urban European population. J Steroid Biochem Mol Biol 2004; 89-90: 621-622.

17. Lardner E, Fitzgibbon M, Wilson S, et al. Hypovitaminosis D in a healthy female population, aged from 40 to 85 years, in the west of Ireland. Ir J Med Sci 2011; 180: 115-119.

18. Hagenau T, Vest R, Gissel TN, et al. Global vitamin D levels in relation to age, gender, skin pigmentation and latitude: an ecologic meta-regression analysis. Osteoporos Int 2009; 20: 133-140.

19. Heaney RP. Barriers to optimizing vitamin D3 intake for the elderly. J Nutr 2006; 136: 1123-1125.

20. Macdonald HM, Mavroeidi A, Barr RJ, et al. Vitamin D status in postmenopausal women living at higher latitudes in the UK in relation to bone health, overweight, sunlight exposure and dietary vitamin D. Bone 2008; 42: 996-1003.

21. Burgaz A, Akesson A, Oster A, et al. Associations of diet, supplement use, and ultraviolet $B$ radiation exposure with vitamin $D$ status in Swedish women during winter. Am J Clin Nutr 2007; 86: 1399-1404.

22. Mensink GB, Fletcher R, Gurinovic M, et al. Mapping low intake of micronutrients across Europe. Br J Nutr 2013; 110: 755-773.

23. Pieniak Z, Verbeke W, Scholderer J. Health-related beliefs and consumer knowledge as determinants of fish consumption. J Hum Nutr Diet 2010; 23: 480-488.

24. Woitge HW, Scheidt-Nave C, Kissling C, et al. Seasonal variation of biochemical indexes of bone turnover: results of a population-based study. J Clin Endocrinol Metab 1998; 83: 68-75.

25. Pasco JA, Henry MJ, Kotowicz MA, et al. Seasonal periodicity of serum vitamin $D$ and parathyroid hormone, bone resorption, and fractures: the Geelong Osteoporosis Study. J Bone Miner Res 2004; 19: 752-758.

26. Darling AL, Hart KH, Gibbs MA, et al. Greater seasonal cycling of 25-hydroxyvitamin $D$ is associated with increased parathyroid hormone and bone resorption. Osteoporos Int 2014; 25: 933-941. 